\title{
Effect of ivacaftor on mucociliary clearance and clinical outcomes in cystic fibrosis patients with G551D-CFTR
}

Scott H. Donaldson, ${ }^{1}$ Beth L. Laube, ${ }^{2}$ Timothy E. Corcoran, ${ }^{3}$ Pradeep Bhambhvani, ${ }^{4}$ Kirby Zeman Agathe Ceppe, ${ }^{1}$ Pamela L. Zeitlin, ${ }^{2}$ Peter J. Mogayzel Jr., ${ }^{2}$ Michael Boyle, ${ }^{5}$ Landon W. Locke, ${ }^{3}$ Michael M. Myerburg, ${ }^{3}$ Joseph M. Pilewski, ${ }^{3}$ Brian Flanagan, ${ }^{4}$ Steven M. Rowe, ${ }^{4}$ and William D. Bennett ${ }^{1}$

'Department of Medicine, University of North Carolina (UNC), Chapel Hill, North Carolina, USA. ²Department of Pediatrics, Johns Hopkins University, Baltimore, Maryland, USA. ${ }^{3}$ Department of Medicine and Bioengineering, University of Pittsburgh, Pittsburgh, Pennsylvania, USA. ${ }^{4}$ Department of Medicine, University of Alabama at Birmingham, Birmingham, Alabama, USA. ${ }^{5}$ Department of Medicine, Johns Hopkins University, Baltimore, Maryland, USA.

BACKGROUND. The ability to restore cystic fibrosis transmembrane regulator (CFTR) function with effective small molecule modulators in patients with cystic fibrosis provides an opportunity to study relationships between CFTR ion channel function, organ level physiology, and clinical outcomes.

Role of funding source: The study sponsor, CFF Therapeutics, had no role in study design, data analysis, or manuscript preparation.

Conflict of interest: All authors report receiving a grant from the Cystic Fibrosis Foundation during the conduct of the study. SHD reports personal fees from AlgiPharma, Sanofi, Ionis, Novartis, and Pulmatrix, grants and personal fees from Galapagos/AbbVie and Nivalis, and grants from Vertex Pharmaceuticals and Parion Sciences. In addition, SHD has a patent "Regulation of Sodium Channels by PLUNC Proteins" (patent no. US 9,549,967 B2). P|M reports grants fom Vertex Pharmaceuticals and Gilead Sciences outside of the submitted work. SMR reports grants from Nivalis, PTC Therapeutics, RaNa, Eloxx, and Celtaxsys; grants and personal fees from Novartis, Sydedgen and Bayer; grants, personal fees, and other nonfinancial support from Vertex Pharmaceuticals and Galapagos/Abbvie; and nonfinancial support from Proteostasis.

License: Copyright 2018, American Society for Clinical Investigation.

Submitted: June 7, 2018

Accepted: November 6, 2018

Published: December 20, 2018

\section{Reference information:}

JCI Insight. 2018;3(24):e122695.

https://doi.org/10.1172/jci.

insight.122695.

METHODS. We performed a multisite, prospective, observational study of ivacaftor, prescribed in patients with the G551D-CFTR mutation. Measurements of lung mucociliary clearance (MCC) were performed before and after treatment initiation ( 1 and 3 months), in parallel with clinical outcome measures.

RESULTS. Marked acceleration in whole lung, central lung, and peripheral lung MCC was observed 1 month after beginning ivacaftor and was sustained at 3 months. Improvements in MCC correlated with improvements in forced expiratory volume in the first second (FEV) but not sweat chloride or symptom scores.

CONCLUSIONS. Restoration of CFTR activity with ivacaftor led to significant improvements in MCC. This physiologic assessment provides a means to characterize future CFTR modulator therapies and may help to predict improvements in lung function.

TRIAL REGISTRATION. ClinicialTrials.gov, NCT01521338.

FUNDING. CFF Therapeutics (COAL11K1).

\section{Introduction}

Small molecule modulators of cystic fibrosis transmembrane regulator (CFTR) function for patients with specific genotypes are making a significant impact in the care of patients with cystic fibrosis $(\mathrm{CF})$ and are dominating current $\mathrm{CF}$ drug development efforts. Appropriately designed phase 3 trials of ivacaftor demonstrated that, in patients with gating mutations, this medication had profound effects on both CFTR function and clinical outcomes $(1,2)$. Prior to US Food and Drug Administration (FDA) approval of this medication, the CF Foundation-Therapeutics Development Network (CF-TDN) designed the G551D Observational Study (GOAL) to test the real-world efficacy of this medication, to collect biospecimens that could be used to identify biomarkers of CFTR function and/or improved health, and to explore the use of specific in vivo bioassays in substudies that may also reflect CFTR function and, thereby, prove useful for future drug development (3).

Mucociliary clearance (MCC), measured by $\gamma$ scintigraphy after the inhalation of radiolabeled particles, has been used extensively in $\mathrm{CF}$ to characterize the consequences of $\mathrm{CF}$ lung disease and to assess the therapeutic potential of inhaled drugs designed to improve the physiologic conditions, such as mucus 
hydration, which govern mucus clearance in vivo. In this study, we tested the hypothesis that CFTR activity plays a central role in controlling the clearance of airway mucus by measuring MCC rates before and after ivacaftor treatment. We also examined the effects of ivacaftor on the CF lung by characterizing treatment effects over time and, in particular, lung regions of interest (ROI) that reflect large and small airway physiologic responses. Finally, by comparing MCC results with other outcome measures, including clinical responses, we examined whether improvements in MCC are related to clinical improvements, which could potentially impact drug development pathways and provide a tool for personalization of treatments. As a multicenter MCC study of a potentially novel therapeutic for CF, this study should also provide insight into the feasibility of using a multisite platform for evaluating new agents using MCC scans in the future.

\section{Results}

\section{Baseline population description}

Twenty-three participants consented to participate at the 4 sites. Twenty-two subjects completed baseline MCC assessments, and 21 completed all assessments. One subject experienced a severe pulmonary exacerbation before the baseline assessment and was excluded from further participation. One subject was excluded from further participation following the baseline MCC scan due to severe noncompliance with the study protocol (Figure 1). Table 1 provides baseline demographic data for the study population that completed at least $1 \mathrm{MCC}$ assessment $(n=22)$.

\section{Clinical response to ivacaftor}

Clinical improvements following ivacaftor were observed in this study and were similar in magnitude to those observed in both randomized controlled studies and the overall GOAL study cohort in this population (2-4). Specifically, significant improvements in lung function, nutritional parameters, and patient reported outcomes were noted, as shown in Table 2 . The clinical response in our study population, therefore, appears to be representative of the typical ivacaftor clinical response in G551D-CFTR patients.

\section{Baseline MCC}

Baseline rates of MCC (AveClr60) were variable across the population but were not significantly different between study sites or subject sexes and did not correlate with baseline lung function (forced expiratory volume in the first second $\left[\mathrm{FEV}_{1}\right]$ percent predicted). However, subjects who were chronically infected with Pseudomonas aeruginosa had significantly slower rates of whole lung (WL) MCC, when compared with those who were either uninfected or intermittently infected (AveClr60 5.5 $\pm 4.9, n=14$; vs. $16.0 \pm 9.4, n=$ $5 ; P=0.005)$. This finding is consistent with those reported in prior studies $(5,6)$.

\section{Effect of ivacaftor on MCC}

WL MCC. Figure 2 shows particle clearance over time in each lung region of interest (ROI). MCC from the whole right lung through 60 minutes (AveClr60) (i.e., before voluntary coughs) was the primary outcome of this study and was previously reported in the main study publication (3). Indeed, WL MCC as indexed by AveClr30, AveClr60, AveClr90, and 24-hour clearance markedly improved after initiating ivacaftor by the 1-month visit and remained at a similarly elevated rate at the 3-month visit (Table 3). Importantly, no significant differences in isotope deposition were detected when comparing baseline and posttreatment central/peripheral $(\mathrm{C} / \mathrm{P})$ deposition ratio and skew of the particle deposition histograms (Table 3). Further, there was no significant correlation between changes in deposition parameters ( $\mathrm{C} / \mathrm{P}$ ratio, skew) and the change in WL clearance. These data provide evidence that the observed acceleration in clearance did not result from a shift in isotope deposition toward the faster-clearing large airways. Examination of individual subject MCC responses, and data trends from individual study sites, also supported the hypothesis that the impact of ivacaftor was robust and not driven by subject outliers or site differences (Figure 3). Finally, the absence of any differences between MCC parameters at 1 and 3 months after ivacaftor initiation supports a rapid ( $<1$ month) and sustained effect that did not wane over time.

Regional assessments of MCC. Examination of regional MCC responses can provide an indicator of the lung compartment (e.g., large vs. small airways) where a therapeutic agent has its greatest impact. The central lung ROI used in this study overlies the lung hilum and, therefore, includes the largest conducting airways. Therefore, analysis of MCC from this ROI provided the opportunity to examine the effect of iva- 


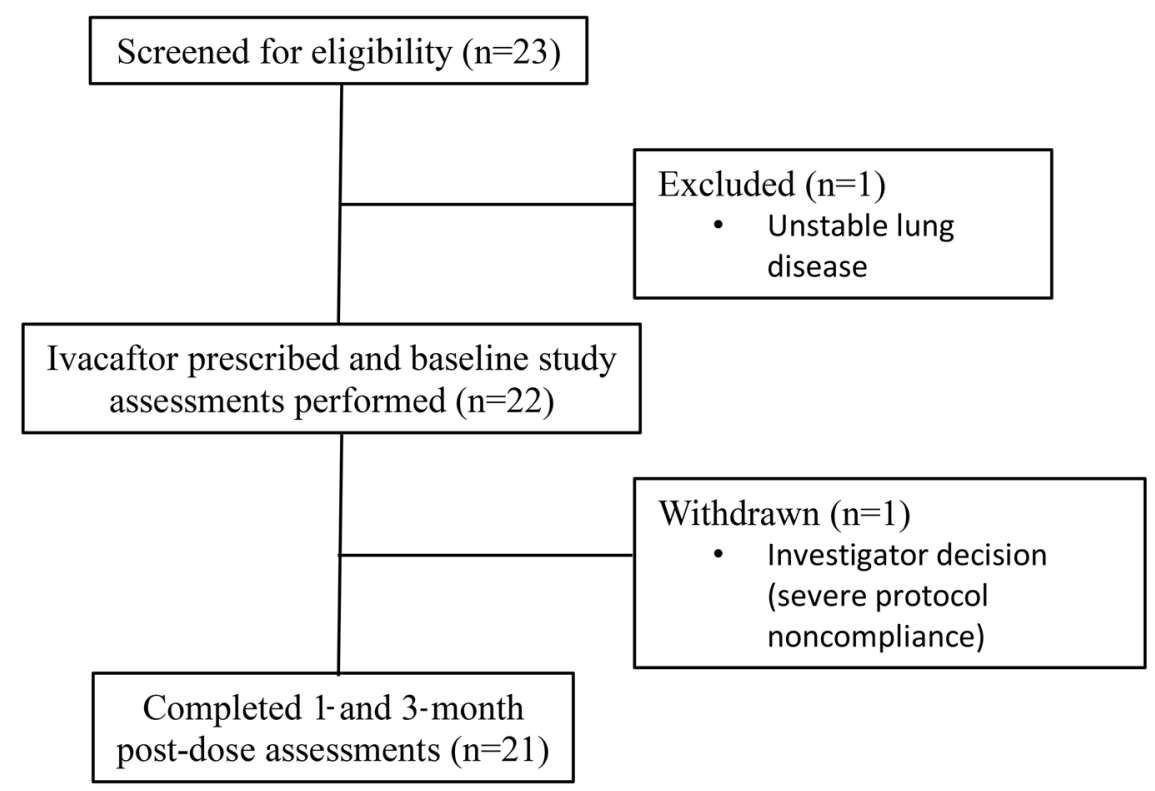

Figure 1. Study flow diagram.

caftor on MCC in a region that is enriched with large airways. Baseline rates of MCC in this compartment were faster than in the WL compartment, given the intrinsically faster rate of clearance from large airways. Following ivacaftor, central lung MCC rates were significantly increased at most time domains (i.e., AveClr30, AveClr60, AveClr90) when compared with baseline (Table 3).

MCC from the peripheral lung region excludes the largest airways located around the lung hilum and provides an assessment of MC that reflects small airway function to a greater degree than WL clearance. We previously showed, using an identical MCC methodology, that MCC is predominantly affected in the peripheral lung in CF when indexed against a group of healthy volunteers (7). In the current study, peripheral lung clearance was negligible at baseline, again consistent with the hypothesis that the small airways are the major site of MCC dysfunction in CF. However, following ivacaftor, a profound ( $~ 7$-fold) increase in peripheral lung MCC was observed (Table 3).

Cough clearance $(C C)$. The ability of cough to clear particles from the lower airways was assessed by having subjects forcefully cough 30 times during the 64- to 90 -minute interval following isotope inhalation. The total number of coughs and peak flow rate during cough maneuvers did not change between baseline and postivacaftor (Vertex Pharmaceuticals) assessments. The percent clearance during this period was numerically increased after starting ivacaftor (Table 3) but not significantly different from baseline $(P=0.22)$. Of note, this method of assessing cough-driven clearance is limited by the fact that the extent of particle clearance preceding the cough interval (i.e., from 0-64 minutes) has the potential to limit the availability of cough-clearable particles (e.g., in central airways vs. distal airways or alveoli). Therefore, the faster rates observed after ivacaftor would create a bias against detecting an improvement in CC. On the other hand, cilia-driven clearance also contributes to the overall clearance observed during the cough interval, and faster postivacaftor rates could account for the numerical (nonsignificant) increases in clearance that were observed during the cough interval without any change in cough-mediated clearance whatsoever.

\section{Predicting MCC responses by baseline characteristics}

We explored whether the observed improvements in MCC in response to ivacaftor were predicted by baseline subject characteristics. In fact, no relationships were observed when examining the potential effects of sex, age, baseline lung function, baseline sweat chloride, or chronic pseudomonas status. The change from baseline WL MCC (AveClr60) was significantly, though weakly, inversely correlated with baseline MCC (Spearman $\mathrm{R}=-0.37 ; P=0.02$ ). These data, therefore, suggest that $\mathrm{MCC}$ responses were similar across 
Table 1. Demographics and baseline characteristics $(n=22)$

\begin{tabular}{|c|c|c|}
\hline Female & $n(\%)$ & $8(34.8)$ \\
\hline Age, years & mean (SD) & $25.2(7.7)$ \\
\hline Pediatric (age $<18$ years) subjects & $n(\%)$ & $4(18.2)$ \\
\hline \multirow[t]{6}{*}{ Genotype Class of non-G551D allele } & I & $4(18.2)$ \\
\hline & II & $14(63.6)$ \\
\hline & III & $1(4.6)$ \\
\hline & IV & $1(4.6)$ \\
\hline & V & $1(4.6)$ \\
\hline & Unidentified & $1(4.6)$ \\
\hline Partially active CFTR mutation & $n(\%)$ & $2(9.1)$ \\
\hline BMI & mean (SD) & $21.9(2.4)$ \\
\hline $\mathrm{FEV}_{1}(\mathrm{I})$ & mean (SD) & $2.67(1.20)$ \\
\hline $\mathrm{FEV}_{1}(\%$ predicted) & mean (SD) & $67.1(24.6)$ \\
\hline FVC $(I)$ & mean (SD) & $3.88(1.22)$ \\
\hline FVC (\% predicted) & mean (SD) & $92.9(17.3)$ \\
\hline Chronic HS use & $n(\%)$ & $12(54.5)$ \\
\hline Chronic dornase alfa use & $\mathrm{n}(\%)$ & $15(68.2)$ \\
\hline
\end{tabular}

patient groups and that patients with the most impaired MCC might be expected to experience the greatest improvements in $\mathrm{MCC}$. Improvements in lung function $\left(\mathrm{FEV}_{1}\right.$ percent predicted) were similarly unrelated to baseline lung function, age, sex, and chronic pseudomonas status.

\section{Correlations between MCC responses and other endpoints}

We next explored whether the observed changes in MCC in response to ivacaftor correlated with clinical outcomes, including lung function $\left(\mathrm{FEV}_{1}\right)$. We employed a mixed model analysis to utilize data from all visits and to incorporate important covariates. In this model, incorporating change in WL AveClr60 and change in $\mathrm{C} / \mathrm{P}$ (an important covariate that often influences $\mathrm{MCC}$ rates), a significant $(P<0.005)$ relationship between the $\mathrm{FEV}_{1}$ and MCC changes was observed. Other potential covariates (e.g., age, sex, baseline

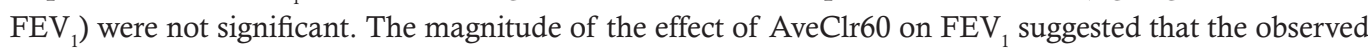
$\sim 10 \%$ absolute change in AveClr60 accounted for a $2.7 \%$ absolute increase in $\mathrm{FEV}_{1}$, or about one-quarter of the total change in lung function. While accelerated MCC did not fully explain the observed improvement in $\mathrm{FEV}_{1}$ in this model, it is perhaps not surprising given the intrinsic variability of these in vivo endpoints, which would be expected to weaken the observed statistical relationship. Further, it is possible that different degrees of MCC improvement could yield similar improvements in $\mathrm{FEV}_{1}$ (i.e., a threshold effect), which would also be expected to weaken the observed statistical relationship. Finally, ivacaftor could yield improvements in $\mathrm{FEV}_{1}$ through mechanisms that are completely independent from MCC. For example, direct smooth muscle and inflammatory cell effects have previously been postulated $(8,9)$

Exploration of other factors that could explain lung function improvements were also explored. The change in central lung, but not peripheral, MCC was similarly related to the change in $\mathrm{FEV}_{1}$. In contrast, as previously reported, changes in sweat chloride did not relate to the change in $\operatorname{FEV}_{1}(10,11)$, nor were other significant relationships observed between changes in MCC parameters and changes in other clinical outcomes (e.g., Cystic Fibrosis Questionnaire - Revised [CFQ-R], Cystic Fibrosis Respiratory Symptom Diary [CFRSD], Sinonasal Outcome Tool [SNOT 20], BMI).

\section{Discussion}

Mutations in CFTR have been known to be the basis of CF lung disease for nearly 3 decades. Two prominent hypotheses link CFTR to mucus transport via the regulation of airway surface liquid (ASL) volume (12) and through bicarbonate secretion (13). Importantly, both of these actions may play a role. Alternatively, CFTR has been linked to lung health via bacterial killing through the regulation of ASL $\mathrm{pH}$ and the activity of antimicrobial substances (14). The approval of ivacaftor as an effective modulator of the G551D-CFTR mutation provided us the opportunity to directly test the hypothesis that CFTR function is tightly linked to MCC. 
Table 2. Change in clinical endpoints

\begin{tabular}{lccc}
\hline & Baseline & 1 month & 3 months \\
FEV $_{\mathbf{1}}(\mathbf{I})$ & $2.67(1.20)$ & $3.07(1.28)^{\mathrm{A}}$ & $3.01(1.28)^{\mathrm{A}}$ \\
FEV $_{\mathbf{1}}$ (\% pred) & $67.1(24.6)$ & $77.5(25.6)^{\mathrm{A}}$ & $75.9(25.1)^{\mathrm{A}}$ \\
Weight (kg) & $63.6(10.8)$ & $65.3(10.9)^{\mathrm{B}}$ & $65.7(10.8)^{\mathrm{B}}$ \\
BMI & $21.9(2.5)$ & $22.5(2.5)^{\mathrm{C}}$ & $22.6(2.2)^{\mathrm{A}}$ \\
Sweat chloride (mEq/I) & $97.5(16.5)$ & $51.0(17.6)^{\mathrm{A}}$ & $47.5(17.5)^{\mathrm{A}}$ \\
CFQ-R resp & $66.9(21.0)$ & $77.5(15.0)^{\mathrm{B}}$ & $81.8(14.4)^{\mathrm{A}}$ \\
CFRSD & $31.6(15.6)$ & $24.2(14.7)^{\mathrm{B}}$ & $22.0(13.6)^{\mathrm{A}}$ \\
SNOT-20 & $1.00(0.67)$ & $0.79(0.76)$ & $0.65(0.66)^{\mathrm{B}}$
\end{tabular}

Mix models were used to assess for changes in each parameter. When significant differences were observed, comparisons to baseline were performed and adjusted using Dunnett's correction. ${ }^{A} P<0.001$; ${ }^{B} P<0.01$; ${ }^{C} P<0.05$ versus baseline. CFQ-R Respiratory Domain, Cystic Fibrosis Questionnaire - Revised, respiratory symptom domain score. A higher score indicates improved respiratory symptoms. CFRSD, Cystic Fibrosis Respiratory Symptom Diary. A lower score indicates improved respiratory symptoms. SNOT-20, Sinonasal Outcome Test. A lower score indicates improved sinonasal symptoms.

Indeed, the large effect on MCC, which was fully realized at the 1-month time point and sustained thereafter, and similarly rapid improvements in clinical outcomes suggest that MCC is intimately linked to CFTR function. This study did not address the mechanism through which CFTR improves MCC, however.

The magnitude of the MCC effect observed after ivacaftor was larger and more predictable (i.e., with fewer nonresponders) than when compared with inhaled therapies such as hypertonic saline (HS) $(7,15)$. Perhaps most notable was the marked increase in peripheral lung clearance observed after ivacaftor (Figure 2C and Table 3). Importantly, peripheral lung clearance was negligible prior to ivacaftor treatment. After ivacaftor, however, robust peripheral lung clearance was observed. This is in agreement with a recently published study that used a pixel-by-pixel analysis of MCC to show that ivacaftor homogeneously increased clearance, regardless of the baseline clearance rate in that pixel (16). We speculate, therefore, that the ability to effectively target distal lung regions via the systemic route provides an important efficacy advantage over inhaled therapies. In addition, ivacaftor's marked impact on MCC and clinical outcomes may also reflect the fact that this drug directly targets CFTR, rather than alternative targets that incompletely substitute for missing CFTR functions. The normalization of peripheral lung clearance and large $\mathrm{FEV}_{1}$ improvement also suggests that, even in these patients with relatively advanced lung disease, a large reversible component exists. Thus, the continued development of systemic therapies and improvements in the delivery of inhaled therapeutics to small airways may be a key component to maximizing treatment responses.

Despite profound improvements in cilia-driven mucus clearance, we saw minimal improvement in CC Although the nature of the MCC assay utilized in this study is not primarily designed to assess CC, and is therefore compromised by the preceding rate of cilia-driven mucus clearance, historical data suggests that a larger fraction of CC should be technically observable (17) but was not. We speculate that the lack of improvement in CC suggests that there are persistent abnormalities in mucus properties that are not corrected by improved CFTR function and the expected improvements in ASL hydration and/or $\mathrm{pH}$. A deeper understanding of the physiologic properties that govern cough- versus cilia-driven clearance could shed light onto mucus properties or mucus-epithelial interactions that should be targeted by other therapeutics (e.g., mucolytics).

Our data demonstrate that measurements of MCC are not only valuable tools for evaluation of CFTR modulator therapies, but that they can be reliably performed on a multisite basis. As the result of careful procedure standardization, the technical quality of images was high and interpretable data was uniformly obtained. Furthermore, between-site differences were not observed in this study, as was also the case in a previous study using these methods at 3 of the involved sites (18). The availability of a multisite MCC testing network may ultimately prove to be an efficient means of demonstrating a relevant physiologic activity within the lung of novel CFTR modulators or other therapeutics designed to improve MCC. Whether or not MCC scans will be sufficiently sensitive to detect MCC changes with less efficacious CFTR modulator regimens, or differentiate modulator regimens with only marginally different activities, is uncertain but is under investigation. 
A

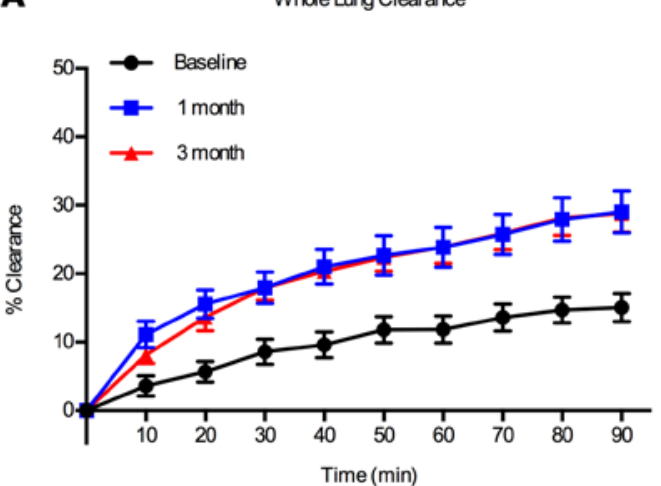

C

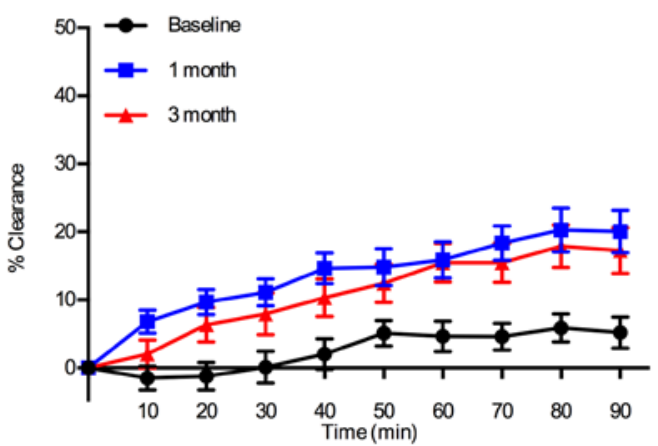

B

Central Lung Clearance

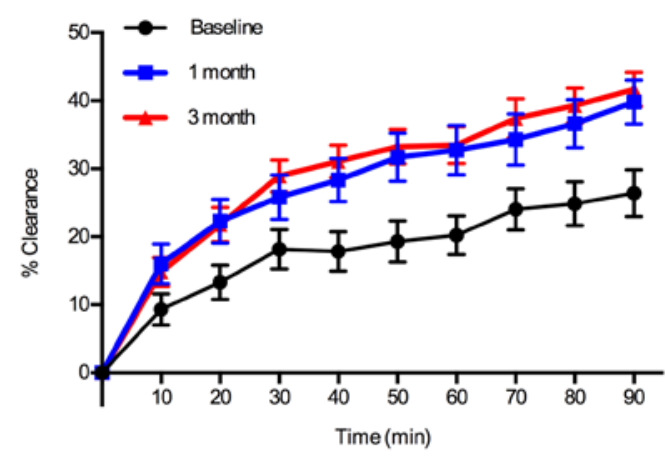

Figure 2. Mean clearance versus time curves. Mean clearance versus time curves ( \pm SEM) in (A) whole lung, (B) central lung, and (C) peripheral lung regions of interest.

Another important question is whether MCC scans can serve as a useful predictor of clinical outcomes. Unlike sweat chloride measurements, or symptom scores, the change in WL and central lung MCC was significantly related to the observed change in $\mathrm{FEV}_{1}$, though it only explained $\sim 25 \%$ of the lung function effect. Although the heterogeneity of CF lung disease is expected to reduce correlations between various physiological endpoints, these data suggest for the first time to our knowledge that improvements in MCC may predict improvements in traditional measures of lung function (i.e., $\mathrm{FEV}_{1}$ ). It is also possible that improvements in MCC may more closely relate to a therapy's impact on pulmonary exacerbations, if indeed these clinical events are related to encroachment of disease into lung regions that previously functioned adequately (i.e., cleared mucus normally). This would be consistent with the apparent paradox observed with HS, where large sustained improvements in MCC and protection against exacerbations has been demonstrated but only small improvements in $\operatorname{FEV}_{1}$ have been observed $(7,19)$.

In summary, ivacaftor treatment provides substantial improvements in CFTR activity in patients with a gating-mutation that translate into profound improvements in $\mathrm{MCC}$ throughout all regions of the $\mathrm{CF}$ lung. MCC changes were robust, not limited to patients with particular characteristics, and were sustained through 3 months of treatment. Changes in whole and central lung clearance were significantly related to changes in $\mathrm{FEV}_{1}$, linking these physiologic endpoints for the first time to our knowledge. Finally, these data show that MCC measurements can be performed well within the context of a multisite study and that these measurements may have value for the early phase assessment of novel CF modulators.

\section{Methods}

A longitudinal, observational cohort study involving 28 centers within the CF-TDN was performed to capture clinical measures and biospecimens (core study) in CF patients age 6 and older with at least 1 copy of the G551D mutation and no prior exposure to ivacaftor (3). The impact of ivacaftor on MCC and the relationship between MCC changes and clinical outcomes was investigated in this nested substudy at 4 centers (UNC, Johns Hopkins University, University of Pittsburgh, and University of Alabama at Birmingham). WL MCC results after 1 month of treatment was previously published with the larger core study findings (3). 
Table 3. Mucociliary clearance parameters

\begin{tabular}{|c|c|c|c|}
\hline MC parameter & Baseline ( $n=22)$ & 1 month $(n=21)$ & 3 month $(n=21)$ \\
\hline Whole lung AveClr30 \% & $5.9(7.5)$ & $14.9(9.5)^{A}$ & $13.2(7.4)^{\mathrm{B}}$ \\
\hline Whole lung AveClr60 \% & $8.5(8.0)$ & $18.7(10.9)^{\mathrm{A}}$ & $17.7(8.1)^{A}$ \\
\hline Whole lung AveClr9o \% & $10.5(8.2)$ & $21.6(11.7)^{A}$ & $21.0(9.0)^{A}$ \\
\hline Whole lung 24-hr Clr \% & 28.7 (19.3) & $36.1(20.6)$ & $46.5(18.1)^{\mathrm{B}}$ \\
\hline Central ROI AveClr30 \% & 13.6 (11.9) & $21.4(14.4)^{\mathrm{c}}$ & $21.9(10.2)^{c}$ \\
\hline Central ROI AveClr60 \% & $16.4(12.6)$ & $26.2(14.9)^{\mathrm{B}}$ & $27.2(9.9)^{B}$ \\
\hline Central ROI AveClr90 \% & $19.3(13.2)$ & $29.7(14.7)^{\mathrm{B}}$ & $31.3(9.7)^{\mathrm{B}}$ \\
\hline Peripheral ROI AveCIr30 \% & $-0.8(9.0)$ & $9.2(7.6)^{\mathrm{B}}$ & $5.4(11.4)$ \\
\hline Peripheral ROI AveClr60 \% & $1.5(8.7)$ & $12.2(9.3)^{\mathrm{B}}$ & $9.1(11.4)^{\mathrm{C}}$ \\
\hline Peripheral ROI AveClr9o \% & $2.8(8.5)$ & $14.6(10.4)^{A}$ & $11.7(12.0)^{\mathrm{B}}$ \\
\hline Cough clearance \% & $3.5(5.7)$ & $6.9(9.3)$ & $6.7(10.2)$ \\
\hline $\mathrm{C} / \mathrm{P}_{\text {norm }}$ ratio & $2.00(0.74)$ & $2.13(0.88)$ & $2.18(0.62)$ \\
\hline Deposition skew & $1.49(0.62)$ & $1.61(0.76)$ & $1.77(0.79)$ \\
\hline
\end{tabular}

Mix models were used to assess for changes in each parameter. When significant differences were observed, comparisons to baseline were performed and adjusted using Dunnett's correction. ${ }^{A} P<0.001 ;{ }^{B} P<0.01 ;{ }^{C} P<0.05$ versus baseline.

Core study. Clinical assessments included spirometry, body weight, sweat chloride analysis, and 3 patient-reported outcome instruments: Cystic Fibrosis Questionnaire-Revised (CFQ-R) (20), Cystic Fibrosis Respiratory Symptom Diary (CFRSD) (21), and Sino-Nasal Outcome Test-20 (SNOT-20) (22). These assessments were conducted at up to 2 baseline visits and then at 1, 3, and 6 months after initiation of ivacaftor. Spirometry was performed according to American Thoracic Society standards (23), and percent predicted values were calculated using reference equations $(24,25)$.

MCC study design. Mucociliary and CC was measured using $\gamma$ scintigraphy at baseline and following 1 and 3 months of ivacaftor therapy at 4 study sites. All sites received on-site training, followed a standard operating procedure (18), and used identical equipment for the delivery of the radioisotope. Subjects were required to withhold use of HS and dornase alfa (Genentech) at least 12 hours before each MCC study and resumed their use only after collection of the final MCC data point (24 hours after inhalation of the radioisotope). Subjects were also required to be clinically stable without new symptoms or pulmonary interventions for 14 days. The change in the average rate of MCC between 0-60 minutes after isotope inhalation (AveClr60) between baseline and 1 month after beginning ivacaftor treatment was the predefined primary outcome.

MCC assessment via $\gamma$ scintigraphy. A standard operating procedure was developed for this study, using methods described previously $(18,26)$. Briefly, a planar $\mathrm{Co}^{57}$ source was used to obtain a transmission scan to delineate the lung contour and establish defined ROI (i.e., peripheral and central lung regions). Technetium-99m-labeled (Tc ${ }^{99 m}$-labeled) sulfur colloid particles were delivered to the subject via DeVilbiss 646 nebulizer, Pulmoaid compressor, and SPIRA dosimeter (0.7-second pulse during inspiration after 100 $\mathrm{ml}$ delay). An electronic flow meter with a visual display and a metronome were used to target a defined inspiratory flow rate (rate $[\mathrm{ml} / \mathrm{s}]=3 \times$ height in $\mathrm{cm}$; rounded to nearest $100 \mathrm{ml} / \mathrm{s}$ ) and breathing pattern (1 second inspiration; 1 second expiration). Serial 2-minute images were then obtained for 64 minutes while encouraging the subject to refrain from coughing. The subject was then asked to forcefully cough through a peak flow device 10 times, every 10 minutes, while continuing to acquire $\gamma$ images for an additional 30 minutes (30 total coughs) to assess CC. The subject returned 24 hours ( \pm 6 hours) after inhaling the radioisotope to assess particle retention at this time point. To avoid the confounding effects of stomach activity on the left lung, particle clearance from the right lung was calculated following correction for isotopic decay. All image analyses were performed at a central site (UNC).

Defining lung ROI and characterizing regional isotope deposition. The 2-dimensional images obtained via $\gamma$ scintigraphy were parsed into WL, C, and P ROI. In this way, the initial pattern of isotope deposition, a factor that could potentially confound the analysis of particle clearance, was assessed. Clearance from the $\mathrm{C}$ and $\mathrm{P}$ regions also was calculated to assess ivacaftor effects in lung regions relatively enriched with large and small airways, respectively. 

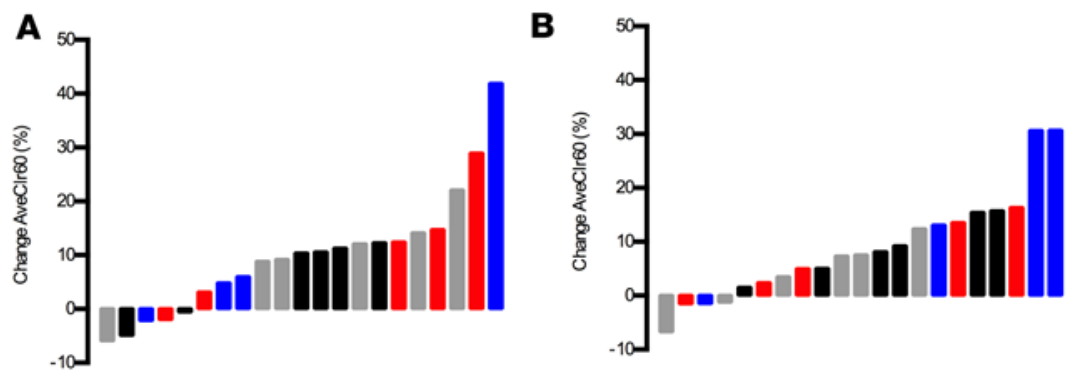

Figure 3. Waterfall plot of the absolute change. Waterfall plot of the absolute change in whole lung AveClr60 from baseline at 1 (A) and 3 (B) months, color coded by study site. Distributions demonstrate robust effect of ivacaftor on MCC that is not driven by site differences.

The $\mathrm{Co}^{57}$ transmission scan was used to identify and outline the WL field and to create $\mathrm{C}$ and P ROI. A rectangular region encompassing the entire right lung was created to define the WL region. A smaller rectangle that included $25 \%$ of the WL area was aligned and centered on the interior boundary of the WL region to define the C ROI (27). The remainder of the lung field was defined the as the P ROI.

Isotope deposition was characterized using a normalized $\mathrm{C} / \mathrm{P}$ ratio $\left(\mathrm{C} / \mathrm{P}_{\text {norm }}\right)$. To do this, the $\mathrm{C} / \mathrm{P}$ ratio, obtained by dividing the number of $\mathrm{TC}^{99 \mathrm{~m}}$ counts on the initial $\gamma$ image following isotope inhalation in the $\mathrm{C}$ and $\mathrm{P}$ regions (i.e., $[\mathrm{C} / \mathrm{P}]_{\mathrm{TC}}$ ), was normalized to tissue attenuation in each $\mathrm{ROI}$ using the $\mathrm{C} / \mathrm{P}$ ratio of $\mathrm{Co}^{57}$ counts on the preceding transmission scan )i.e., $[\mathrm{C} / \mathrm{P}]_{\text {trans }}$ :

$(\mathrm{C} / \mathrm{P})_{\mathrm{Tc}} /(\mathrm{C} / \mathrm{P})_{\text {trans }}=\mathrm{C} / \mathrm{P}_{\text {norm }}$.

The relative intensity of signal on a transmission image has been shown to be a useful surrogate for that region's lung volume (as reported by Xenon equilibrium scans) when normalizing C/P ratios (27). The resulting normalized $\mathrm{C} / \mathrm{P}$ ratio provides an index of relative deposition in the central and peripheral lung regions that may then be used to assess deposition patterns at different study visits. Particle deposition is also used as a covariate in MCC analyses because MCC tends to be faster in central airways than in peripheral airways. As an independent assessment of deposition pattern heterogeneity, a histogram of radioactive counts in each pixel of the initial deposition in the whole right lung image following $\mathrm{Tc}^{99 \mathrm{~m}}$ inhalation was constructed, and the skew of its distribution was calculated (28).

Analysis of regional MCC and CC. The retention (Rt)of radioactive counts in each ROI (WL, C, P) at sequential time points was corrected for isotopic decay and background radiation and was then expressed as a fraction of the initial counts. Percent clearance values were calculated from these retention values: $100 \times(1-\mathrm{Rt})$. Percent clearance at designated 10-minute intervals throughout the 94-minute period was determined using the sum of two 2-minute images that followed each 10-minute interval (Figure 2). Twenty-four-hour clearance values were obtained using a 30-minute image at this time point. To describe each clearance vs. time data set, the average percent clearance over the first 30-, 60-, and 90-minute periods of observation were computed using each 10-minute data point. These computed values - identified as AveClr30, AveClr60, and AveClr90, respectively - are proportional to the AUC and represent the average clearance at the midpoint of each period of clearance vs. time observation (18).

Radioisotope clearance between 64 and 94 minutes of the MCC scan reflects the combined effects of cilia and CC. Because the preceding period of isotope clearance (0-64 minutes) determines the amount of tracer available to be cleared during this CC period, the Rt value between 60 and 90 minutes was normalized for each scan to the same starting point:

$\mathrm{CC}=100 \times\left(\left[1-\left(\mathrm{Rt} / \mathrm{Rt}_{60 \mathrm{~min}}\right]\right)\right.$.

The total number (voluntary + spontaneous) and force of coughs (peak flow of every fifth effort) were recorded to allow assessment of these potential confounders.

Statistics. Mixed models were used to compare MCC parameters, deposition indices, and clinical outcomes measured at baseline and after ivacaftor (1 and 3 months) using "visit" as the fixed effect and "subject" as the random effect. Comparisons to baseline were performed and adjusted using Dunnett's correction. $P$ values below 0.05 were considered significant. Similarly, mixed models (exploratory variable and visit as fixed effects; subject as random effect) were used to assess the effect of clinical parameters (e.g., baseline $\mathrm{FEV}_{1}$, sex, chronic pseudomonas infection status) on baseline MCC parameters and MCC 
responses to ivacaftor. Finally, mixed models were also used to examine the relationship between measured changes in MCC and clinical outcomes (e.g., lung function). The significance of relationships between individual variables was tested using nonparametric Spearman's rank correlation analysis. All values are expressed as the mean \pm SD unless noted otherwise. Statistical calculations were performed using JMP Pro v13.0 (SAS Institute Inc.).

Study approval. All participants (and their guardians, as applicable) provided written informed consent, and the study was approved by IRBs at University of North Carolina at Chapel Hill, Johns Hopkins School of Medicine, University of Pittsburgh School of Medicine, and University of Alabama at Birmingham School of Medicine (ClinicialTrials.gov, NCT01521338).

\section{Author contributions}

SHD, SMR, and WDB led the study design. SHD, BLL, TEC, PB, KZ, AC, PLZ, PJM, MB, LWL, MMM, JMP, BF, SMR, and WDB contributed to data collection, and data interpretation for this manuscript. SHD, $\mathrm{KZ}, \mathrm{AC}$, and WDB performed analyses. SHD and WBD drafted the manuscript. BLL, TEC, KZ, AC, PLZ, and SMR also wrote and edited the manuscript.

\section{Acknowledgments}

This work was supported by the CFF Therapeutics, with support from P30 DK065988 at UNC; P30 DK072482 and UL1TR001417 at UAB; UL1TR001079 at Johns Hopkins University; and P30 DK072506 and UL 1RR024153 at University of Pittsburgh. The investigators would like to acknowledge the Therapeutics Development Network Coordinating Center (Seattle, Washington, USA), for their invaluable assistance and Sonya Heltshe for helpful discussions. Finally, the authors wish to acknowledge the contributions of patients and families who participated in this study, as well as research coordinators and technicians who contributed to this work.

Address correspondence to: Scott H. Donaldson, 300 Meadowmont Village Circe, Suite 204, Chapel Hill, North Carolina 27517, USA. Phone: 984.974.9198; Email: Scott_Donaldson@med.unc.edu.

PLZ's present address is: Department of Pediatrics, National Jewish Health, Denver, Colorado, USA.

LWL's present address is: Department of Medicine, Ohio State University, Columbus, Ohio, USA.

1. De Boeck K, et al. Efficacy and safety of ivacaftor in patients with cystic fibrosis and a non-G551D gating mutation. $J$ Cyst Fibros. 2014;13(6):674-680.

2. Ramsey BW, et al. A CFTR potentiator in patients with cystic fibrosis and the G551D mutation. N Engl J Med. 2011;365(18):1663-1672.

3. Rowe SM, et al. Clinical mechanism of the cystic fibrosis transmembrane conductance regulator potentiator ivacaftor in G551D-mediated cystic fibrosis. Am J Respir Crit Care Med. 2014;190(2):175-184

4. Davies JC, et al. Efficacy and safety of ivacaftor in patients aged 6 to 11 years with cystic fibrosis with a G551D mutation. Am J Respir Crit Care Med. 2013;187(11):1219-1225.

5. Laube BL, Sharpless G, Benson J, Carson KA, Mogayzel PJ. Mucus removal is impaired in children with cystic fibrosis who have been infected by Pseudomonas aeruginosa. J Pediatr. 2014;164(4):839-845.

6. Locke LW, et al. Pseudomonas infection and mucociliary and absorptive clearance in the cystic fibrosis lung. Eur Respir J. 2016;47(5):1392-1401.

7. Donaldson SH, Bennett WD, Zeman KL, Knowles MR, Tarran R, Boucher RC. Mucus clearance and lung function in cystic fibrosis with hypertonic saline. NEngl J Med. 2006;354(3):241-250.

8. Cook DP, et al. Cystic Fibrosis Transmembrane Conductance Regulator in Sarcoplasmic Reticulum of Airway Smooth Muscle. Implications for Airway Contractility. Am J Respir Crit Care Med. 2016;193(4):417-426.

9. Pohl K, et al. A neutrophil intrinsic impairment affecting Rab27a and degranulation in cystic fibrosis is corrected by CFTR potentiator therapy. Blood. 2014;124(7):999-1009.

10. Durmowicz AG, Witzmann KA, Rosebraugh CJ, Chowdhury BA. Change in sweat chloride as a clinical end point in cystic fibrosis clinical trials: the ivacaftor experience. Chest. 2013;143(1):14-18.

11. Barry PJ, Jones AM, Webb AK, Horsley AR. Sweat chloride is not a useful marker of clinical response to Ivacaftor. Thorax. 2014;69(6):586-587.

12. Boucher RC. Evidence for airway surface dehydration as the initiating event in CF airway disease. J Intern Med. 2007;261(1):5-16

13. Quinton PM. Cystic fibrosis: impaired bicarbonate secretion and mucoviscidosis. Lancet. 2008;372(9636):415-417.

14. Pezzulo AA, et al. Reduced airway surface $\mathrm{pH}$ impairs bacterial killing in the porcine cystic fibrosis lung. Nature. 2012;487(7405):109-113.

15. Trimble AT, et al. Hypertonic saline has a prolonged effect on mucociliary clearance in adults with cystic fibrosis. J Cyst Fibros. 
2018;17(5):650-656

16. Bennett WD, et al. Homogeneity of Aerosol Deposition and Mucociliary Clearance are Improved Following Ivacaftor Treatment in Cystic Fibrosis. J Aerosol Med Pulm Drug Deliv. 2018;31(4):204-211.

17. Bennett W, Zeman K, Donaldson SH, Donohue JF, Knowles MR, Boucher RC. Cough Clearance is Less Effective in Cystic Fibrosis than Chronic Bronchitis. Am J Resp Crit Care Med. 2004;169(7):A386.

18. Bennett WD, et al. Multisite comparison of mucociliary and cough clearance measures using standardized methods. $J$ Aerosol Med Pulm Drug Deliv. 2013;26(3):157-164.

19. Elkins MR, et al. A controlled trial of long-term inhaled hypertonic saline in patients with cystic fibrosis. $N E n g l J M e d$. 2006;354(3):229-240.

20. Quittner AL, Buu A, Messer MA, Modi AC, Watrous M. Development and validation of The Cystic Fibrosis Questionnaire in the United States: a health-related quality-of-life measure for cystic fibrosis. Chest. 2005;128(4):2347-2354.

21. Goss CH, Edwards TC, Ramsey BW, Aitken ML, Patrick DL. Patient-reported respiratory symptoms in cystic fibrosis. J Cyst Fibros. 2009;8(4):245-252.

22. Piccirillo JF, Merritt MG, Richards ML. Psychometric and clinimetric validity of the 20-Item Sino-Nasal Outcome Test (SNOT20). Otolaryngol Head Neck Surg. 2002;126(1):41-47.

23. [No authors listed]. Standardization of Spirometry, 1994 Update. American Thoracic Society. Am J Respir Crit Care Med. 1995;152(3):1107-1136.

24. Hankinson JL, Odencrantz JR, Fedan KB. Spirometric reference values from a sample of the general U.S. population. Am J Respir Crit Care Med. 1999;159(1):179-187.

25. Wang X, Dockery DW, Wypij D, Fay ME, Ferris BG. Pulmonary function between 6 and 18 years of age. Pediatr Pulmonol. 1993;15(2):75-88

26. Donaldson SH, Corcoran TE, Laube BL, Bennett WD. Mucociliary clearance as an outcome measure for cystic fibrosis clinical research. Proc Am Thorac Soc. 2007;4(4):399-405.

27. Zeman KL, Wu J, Donaldson SH, Bennett WD. Comparison of 133 xenon ventilation equilibrium scan (XV) and $99 \mathrm{~m}$ technetium transmission (TT) scan for use in regional lung analysis by 2D gamma scintigraphy in healthy and cystic fibrosis lungs. $J$ Aerosol Med Pulm Drug Deliv. 2013;26(2):94-100.

28. Bennett WD, Xie M, Zeman K, Hurd H, Donaldson S. Heterogeneity of Particle Deposition by Pixel Analysis of 2D Gamma Scintigraphy Images. J Aerosol Med Pulm Drug Deliv. 2015;28(3):211-218 